\begin{tabular}{|l|l|}
\hline FM-1 & 341 \\
\hline
\end{tabular}



MILITARY TECHNICAL COLLEGE

CAIRO - EGYPT

\title{
EFFECT OF OIL LEVEL ON HYDRAULIC SYSTEM PERFORMANCE
}
Ibrahim SALEH ${ }^{*}$
S. ELSHAMARKA
A. KHATTAB

\section{ABSTRACT}

Oil level in higdraulic reservoir has been varied to study its undesirable effect on oil temperature. The study shows that the initial air cushion volume as well as its fluctuations, affect the pressure inside; thus arrtion, cavitation and heat and mass transfer would be infkuenced. These conditions play the main role in optimizing the oil level in the reservoirs.

Taking into consideration the other working conditions Idischarges, temperatures, oil properties...etc.l, it was found that both high and law oil level would increase the working oil temperature.

\section{INT RODUCTION}

Hydraulic equipments have recently become so influential in the design of industrial machinery, that without hydraulies gutmation known nowedays would have not been possible [1]. Hydraulic power systems consist mainly of pumps, motors, cylinders, control valves and accessories. The term "ACCESSORIES" actually leads to a false impression, when considering their importance in system performance and safety. One of the very basic accessories is the oil tank in open circuits. Oil tank has a number of functions that affect widely the hydraulic circuit performance, among which - this paper is concerned with its work as; heat exchanger and air separator. To fulfill these functions the tank size is chosen ( 3 to 5 ) times the pump flow rate per minute, with (10 to $15 \%$ ) lof its volume as an air cushion[1]. An air breather with suitable filter is usually provided to allow the

* Assistant prof. ; Chair of mechanical power and energy, M.T.C.;Cairo. * Lecturer; Chair of mechanical power and energy; M.T.C.; Cairo, Egypt. 


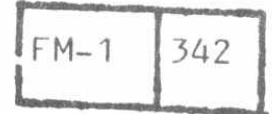

oil level fluctuation in a dust-proof space.

In some engineering applications, severe fluctuations of oil level are observed. For such cases, oil temperature increases for both lower and higher oil level than prescribed. The objective of this paper is to investigate these effects.

\section{EFFECT OF OIL LEVEL}

Oil level in the tank has a direct impact on the pump suction conditions as it usually causes an increase in oil temperature. This may be explained by :

- Decreasing the thermal capacity of the system reservoir.

- Increasing the probability of cavitation in the pump suction side.

- Promoting the formation of free vortex near the suction pipe, that increases the air entrainment from the atmosphere.

- Increasing the dissolved air liberation in the pump suction, which affects the pump volumetric efficiency.

- Increasing leakage due to lower oil viscosity in effect of higher temperatures.

- In addition to that, lower oil level promotes the suction of solid deposits, thus deterioration of both the pump all-together with the system service life.

To explain the temperature increase as the oil level gets higher, one should investigate its effect on:

- The rate of heat generation (hydraulic losses) in the oil.

- The rate of heat dissipation from the tank (cooling effect).

\section{BASIC EQUATION GOVERNING THE PRESSURE VARIATION INSIDE.}

The difference between input and output powers of the hydraulic system represents the heat delivered to the oil (losses). This energy is dissipated to the surroundings through the elements of the system, (oil tank and heat exchanger if found). The pumping volumetric efficiency has a direct impact on the over-all system losses.

For certain operating conditions, the effect of oil level on heat 
generation rate may be investigated by evaluating the air pressure fluctuations above the oil in the tank. This pressure fluctuation has shown considerable contribution as to heat generation and dissipation.

For the oil tank given in $f i g .1$, the continuity equation applied on the oil inside the tank, when neglecting the compressibility effect gives:

$$
Q_{n}=Q_{0}-Q_{i}=A_{T} \cdot \frac{d y}{d t}{ }^{\star}
$$

While for air enclosed above the oil in the tank, it gives: [2]

$$
Q_{a}=A_{T} \cdot \frac{d y}{d t}+\left(\frac{V_{a O}+A_{T} \cdot y}{B}\right) \cdot \frac{d p}{d t}
$$

where: $Q_{a}=C_{d} \cdot A_{n} \sqrt{2\left(p_{o}-p\right) / \rho}$

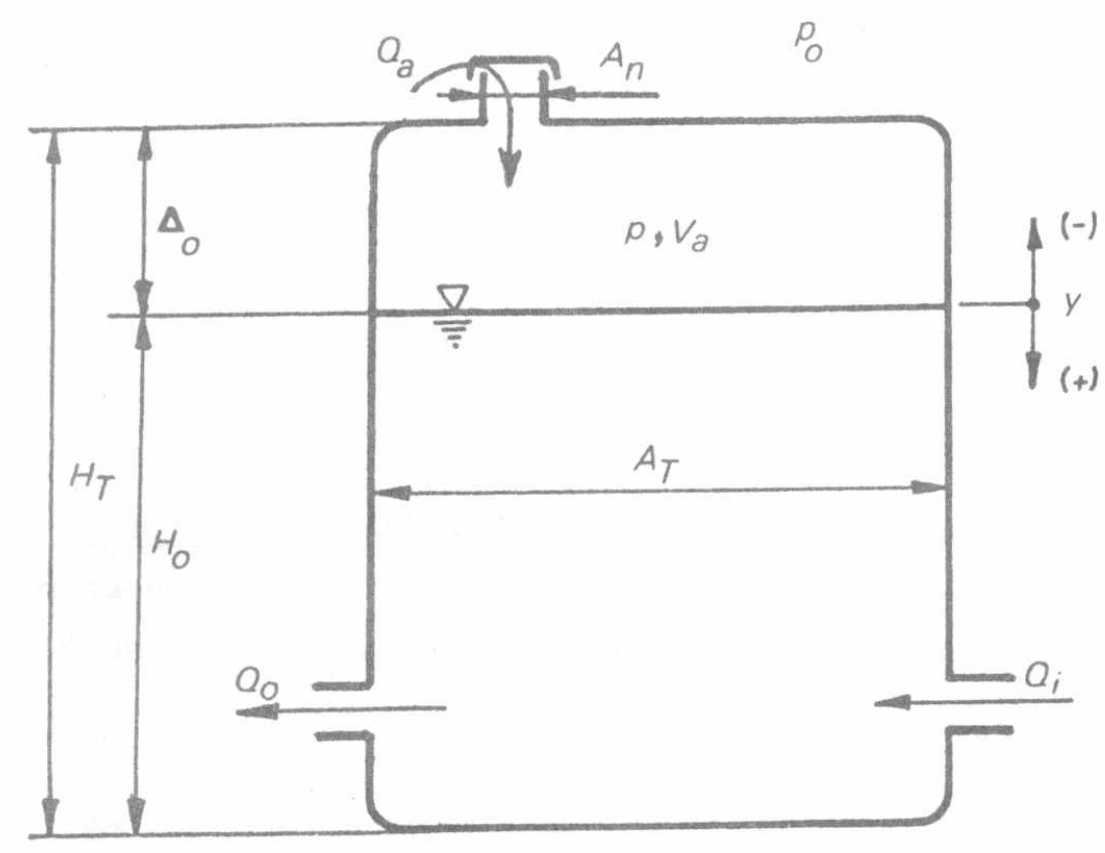

Fig. 1. Basic parameters applied on the reservoir

* All the symbols are listed at the end of the paper. 
From equations (1), (2) and (3):

$$
C_{d} \cdot A_{n} \sqrt{\frac{2\left(p_{0}-p\right)}{\rho}}=Q_{n}+\frac{\left(V_{a O}+A_{T} \cdot y\right)}{n \cdot p} \cdot \frac{d p}{d t}
$$

For specified flow conditions, and initial oil level, the differential equation (4) representing the air pressure $p$ inside the tank can be solved numerically as function of time.



Fig.2. Time responoe of the reservoir system pressure

EFFECT OF THE INITIAL DEGREE OF FILLING OF OIL TANK [V OO OI H ] Basic differential equation (4) shows that according to the operating net flow rate $Q_{n}$ of the oil, the pressure $p$ inside the tank varies such that it approaches assymptotically the value $p_{s}$ given by:

$$
p_{S}=p_{0}-\frac{\rho_{\cdot} Q_{n}^{2}}{2 C_{d}^{2} \cdot A_{n}^{2}}
$$

with variable course of variation of time constant $\tau$ calculated as follows:

$$
\frac{-\Delta p_{0}}{\tau}=\frac{d p}{d t}(0)
$$


from which: $\quad T=\frac{\rho \cdot V_{a o} \cdot Q_{n}}{2 C_{d}^{2} \cdot A_{n}^{2} \cdot n \cdot p_{o}}$

as :

$V_{\text {ao }}={ }_{T}^{A_{T}} \cdot \Delta_{0}=A_{T} \cdot\left(H_{T}-H_{0}\right)$

the

$$
\tau=\frac{\rho \cdot Q_{n} \cdot A_{T}\left(H_{T}-H_{0}\right)}{2 C_{d}^{2} \cdot A_{n}^{2} \cdot n \cdot p_{O}}
$$
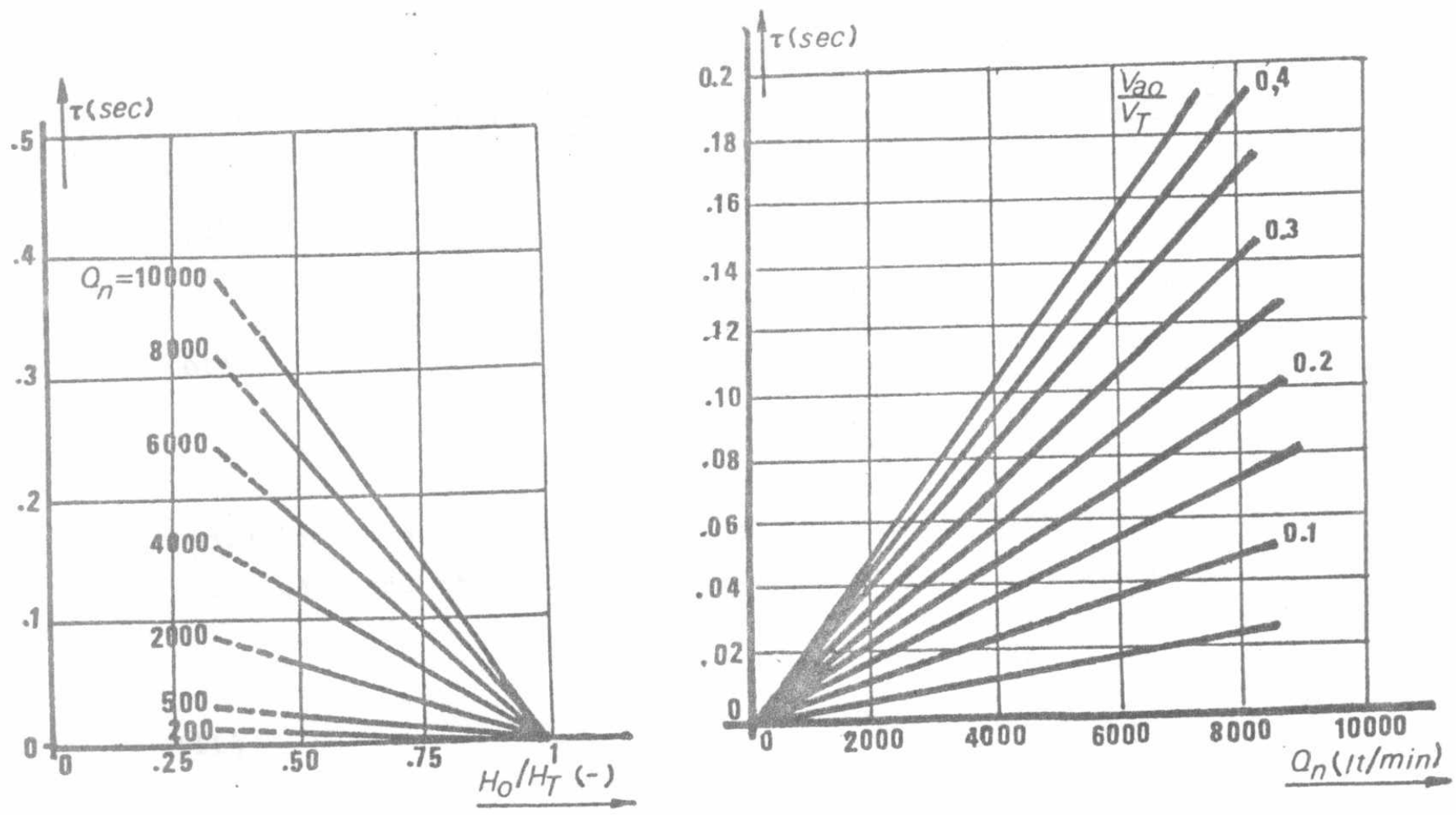

Fig.3. Effect of the degree of filling on the time constant

VOLUMETRIC EFFICIENCY DECREASE AND HEAT GENERATION

Reducing the pressure above the oil in the tank by $\Delta p_{m}$ i decreases the air solubility by $\Delta \epsilon_{a}$. [3]

$$
\Delta \epsilon_{a}=-K \cdot \Delta p_{m}
$$

where $K$ is a constant depending on the oil. 




Fig. 4. The solubility of air in various liquids

$\Delta p_{m}=\Delta p_{0}\left(1-\frac{\tau}{T_{1}}\left(1-e^{-T_{1} / \tau}\right)\right.$

where:

$$
\Delta_{p_{0}}=p_{0}-p_{S}=\frac{\rho Q_{n}^{2}}{2 C_{d}^{2} \cdot A_{n}^{2}}
$$

For the vapour bubbles liberation during cavitation due to pressure drop, and by analogy, defining the ratio of its volume to the total oil by $\Delta \epsilon_{V}$, it may be assumed as:

$$
\Delta \epsilon_{V}=-k_{1}\left(p_{m}-p_{C r}\right)^{n l}
$$

where $K_{1}$ and $n_{1}$ are to be found experimentally.

From equations (9) and (11), the total air and bubbles volumetric ratio to the working oil may be given by:

$$
\Delta \epsilon_{T}=\Delta \epsilon_{a}+\Delta \epsilon_{V}
$$

In this case, the volumetric efficiency $\eta_{v o}$ will be reduced to : $\eta_{v}$ given by:

$$
\eta_{v}=\eta_{v o}\left(1-\Delta \epsilon_{T}\right)
$$


The rate of heat generation $P_{G}$ will be increased by $\Delta P_{G}$ given by:

$$
\Delta P_{G}=\left(\frac{\eta_{V O}-\eta_{V}}{\eta_{V O}}\right) P_{i n p}=\Delta \epsilon_{T} \cdot P_{\text {inp }}
$$

where:

$$
P_{G}=\left(1-\eta_{0}\right) P_{i n p}
$$

\section{AT DISSIPATION FROM THE TANK}

The heat transfer from the system reservoir takes place in two different ways:

- heat transfer through the tank walls.

- latent heat carried by oil vapour diffused in the air on top of the oil level.

Convection mode plays the major role in sensible heat transmission across the walls. On the other hand itis believed that thermal resistance of air film arround the tank is predominant. In practice an overall heat transfer coefficient $U\left(5: 20 \mathrm{~W} / \mathrm{m}^{2} . C^{0}\right)$ is traced [4] and the sensible heat flux $q_{S}$ is given by:

$$
q_{s}=U \cdot \Delta T
$$

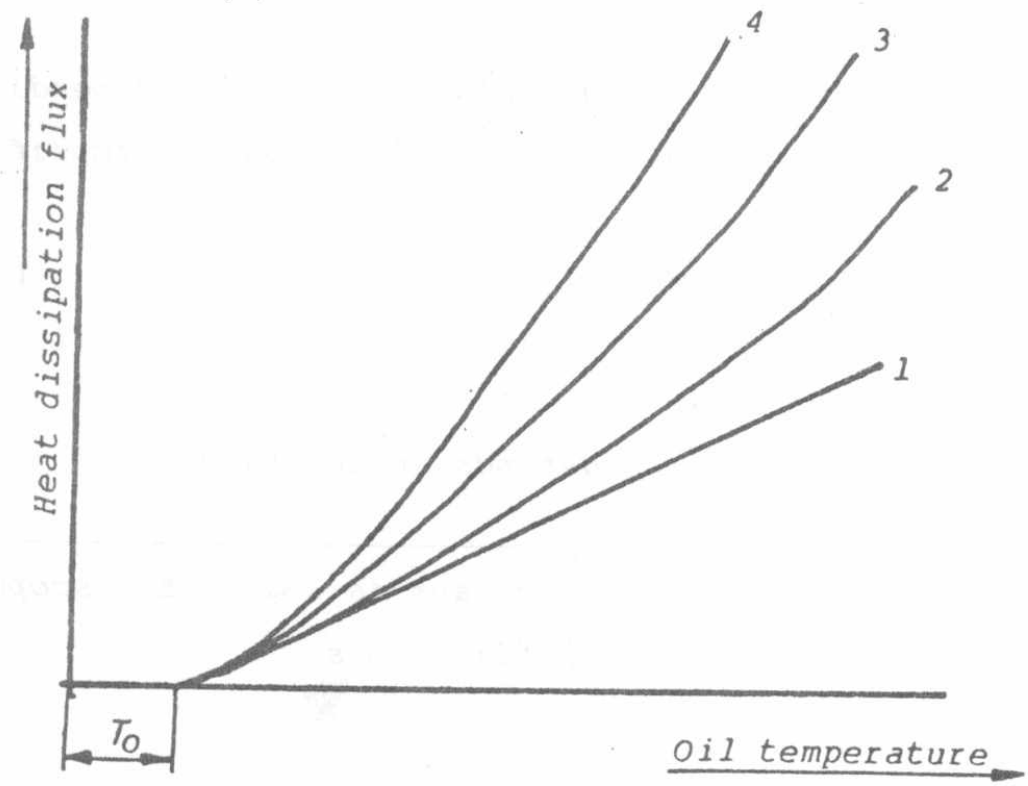

Fig.5. Typical heat dissipation flux 
Line (1) in figure 5 represents that equation, and shows the dependence of $q_{S}$ on the oil temperature.

The mass transfer within the oil tank enclosure depends on the regime of air motion inside. This regime will be handled in the first approximation as stagnant conditions with stationary oil level. Thus the mass flux of oil vapour across the area of interface is given by:

$$
m_{0}^{\prime}=-D P \cdot \frac{d W}{d \Delta_{0}}+W \rho V_{S}
$$

Although air is assumed stagnant, its bulk will have certain velocity $v_{s}$ [in last equation] away of the surface. This velocity is determined utilizing the stagnation condition.

Taking the fluctuations of the oil level into consideration, accounts for an increase of the velocity of the air bulk by $v_{C}{ }$ This velocity has been estimated through a computer program based on the mathematical model representing the air motion due to the fluctuations of the oil level in the tank. Such effect showed some 100 times the effect of molecular diffusion. Finally a worked out formula of the total latent heat $f$ lux can be written as:

$$
q_{1}=C_{1} \cdot \frac{A^{\prime}}{A} \cdot \frac{D}{\Delta_{0}} \cdot \rho \cdot \frac{p_{v}}{p_{m}-p_{v}}\left[1+\frac{\Delta_{0}}{2 D}\left(v_{s}+v_{C}\right)\right] \cdot L_{v}
$$

Further numerical approximation would probably be useful depending on the rough estimated value of $v_{C}^{*}$, and equation (18) may be simplified as:

$$
q_{1}=C_{2} \cdot \frac{A^{\prime}}{A} \cdot \rho \cdot \frac{p_{V}}{p_{m}-p_{V}} \cdot V_{C} \cdot L_{V}
$$

The constant $C_{2}$ in the last equation depends on the type

Detailed study of air motion inside the tank is beyond the scope of work and will be continued in later publications. 
of used oil, and average oil concentration in the bulk of the air (between the two values of equilibrium concentration in the interface area, and zero oil concentration at the top of the tank). The ratio $A^{\prime} / A$ relates $q_{I}$ to the surface area of ofl tank $\mathrm{A}$ Instead of the area of interface $A^{\prime}$.

Equation 19 is then represented by the exponential form 1 ines $(2,3$ \&4) foi fferent total air pressures $p_{2}>p_{3}>p_{4}$ shown in figure (5).

llustrative case study was conducted on $50 \%$ ethylene waterglycol $\operatorname{mix}$ and results are shown in figure (6).

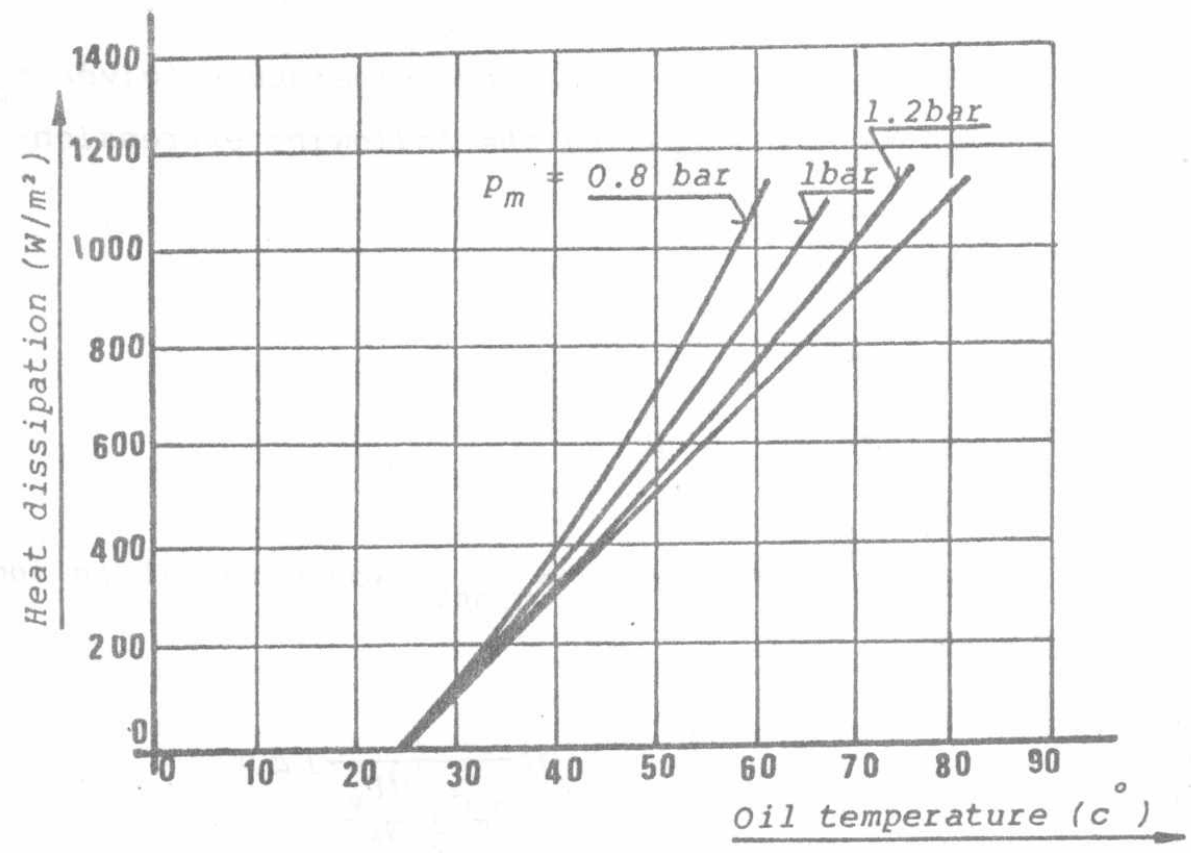

Fig.6. Heat dissipation determined for the case study

The fluctuations of oil level has an effect on the latent heat $f$ lux as follows: when the oil level drops down during active strokes, the total air pressure inside the tank is reduced as previously shbwn, thus accounting for higher equilibrium concentration of oil vapour into air, hence increased flux. On the other hand, oil level fluctuations increase the velocity of the air bulk which plays an important role in mass transfer.

It must be noticed that the type of oil (i.e vapour pressure characteristics) is the most significant factor in the whole process and for oils of low vapour pressure, the latent heat flux may be ignored, while for oils of high $p_{V}$ (e.g waterglycols) such effect is appreciable. 


\section{DETERMINATION OF THE OPTIMUM DEGREE OF FILLING}

For the steady state conditions and from equations (14) and (19):

$$
\Delta \epsilon_{T} \cdot P_{i n p}=C_{3} \cdot \frac{p_{v}}{P_{m}-p_{v}}
$$

where:

$$
C_{3}=C_{2} \cdot \rho \cdot v_{C} \cdot L_{V} \cdot A
$$

It must be noticed that $\Delta \epsilon_{T}$ given in the last equation is function of $p_{m}$ and temperature.

Solving this equation for given operating temperature (given $p_{v}$ ), the optimum value of $p_{m}$ can be calculated by the following expression:

$$
p_{m . o p t .}=p_{v}+\sqrt{\frac{c_{3} \cdot p_{v}}{p_{i n p} \cdot k}} \text { * }
$$

$$
\text { for : } \quad p_{0}>p_{m}>p_{s}
$$

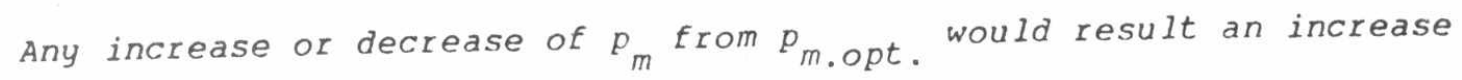
in temperature given by :

$$
\Delta T=\left[K \cdot P_{i n p} \cdot\left(p_{m}-p_{v}\right)^{2}-c_{3} p_{v}\right]\left[\frac{1}{p_{m} c_{3}\left(\frac{\left.d p_{v}\right)}{d t}\right] \Delta p_{m o}}\right.
$$

Refering to equations (7), (9) and (10) and as:

$$
p_{m}=p_{0}-\Delta p_{m}
$$

$p_{m}$ is found to be function of both $Q_{n}$ and $H$.

For given working conditions, ambient temperature and vapour pressure; if $Q_{n}$ is taken to be constant, the increase of temperature may be given

Equation (21) is calculated for the case neglecting the effect of vapour bubbles liberation $\left(\Delta \epsilon_{v}\right)$. 
as function of the variation of oil optimum initial height in the tank ( $H_{0}$ ) as follows:

$$
\Delta T=\frac{\Delta p_{0}^{2} \cdot A_{T}\left[K \cdot P_{i n p} \frac{-C_{3} \cdot p_{V}}{\left(p_{m}-p_{v}\right)^{2}}\right] \cdot\left[1-e^{-T 1 / \tau}\left(1+\frac{T}{\tau}\right)\right] \cdot \Delta H_{0}}{Q_{n} \cdot p_{b} n \cdot T_{1}\left[C_{3} \cdot p_{m} \cdot d p_{v} / d t+A_{T} U\right]}
$$

From which the optimum height of oil may be determined by putting $(T=0)$

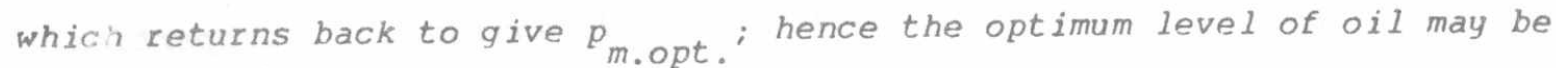
also evaluated.



Fig.7. Chart of calculation of the optimum height of oil in the tank.

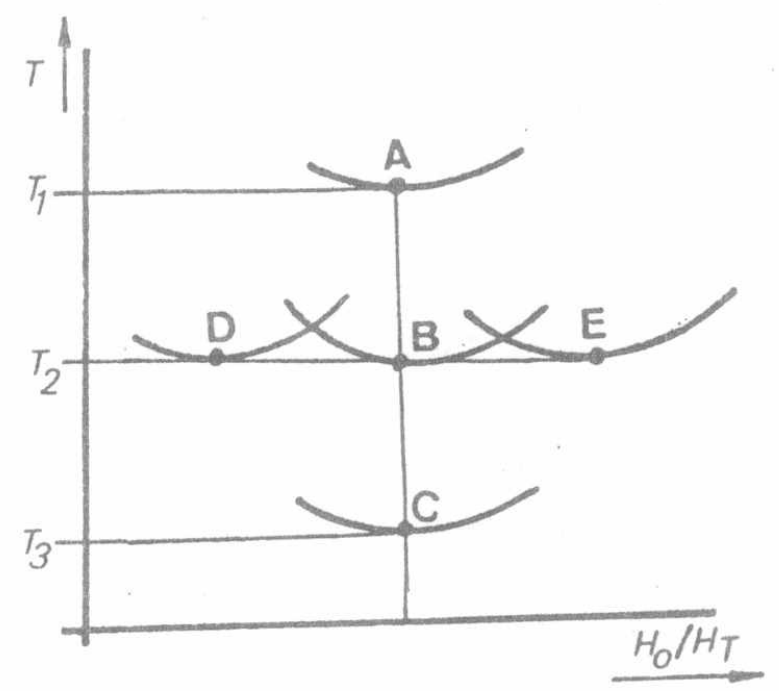

Fig.8. Variation of temperature with other heights than optimum

Case study was developped to. show that the temperature increase if oil level is changed from its optimum value as shown in figure (8). 
Figure 7 shows an example of the final chart, indicating the procedure of determination of the optimum oil level height if given the temperature of working oil and the operating rate of discharge.

\section{CONCLUSTON}

For certain operating conditions.(working power, discharges...etc.) with certain design parameters (construction, dimensions, oil properties...etc.) an optimum level height may be evaluated. If the oil level alters from its optimum value (decreases or increases) the working oil temperature increases. This will be useful in both design and working phases in open hydraulic circuits,

\section{REFERENCES}

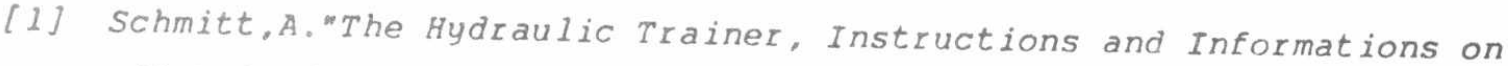
all Hydaulics ",REXROTH,W.Germany.

[2] GUILLON, M."Hydraulic Servomechanisms, Analysis and Design;(Plenum Press New POFB-1969)

[3] A.KHATTAB. "Modelling and Analysis of Performance of Aircraft Hydraulic servo-unit" thesis of M.SC.,M.T.C.;Cairo,Egypt.

[4] Perry: "Chemical Engineer's Handbook"; 5th ed.,pp10.37 to 10.70.

[5] ASHRAE, "Handbook of Fundumentals"; 1983, MCGraw Hill.

\section{NOMENCLATURE}

A

$A^{\prime}$

${ }^{A}$

${ }^{A} T$

B

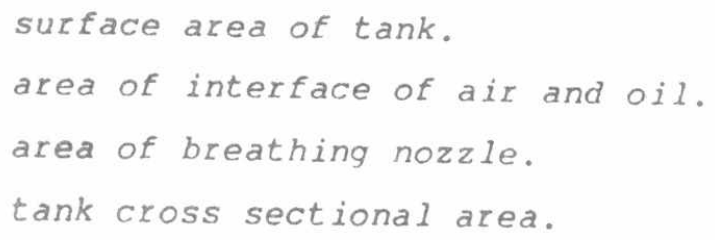









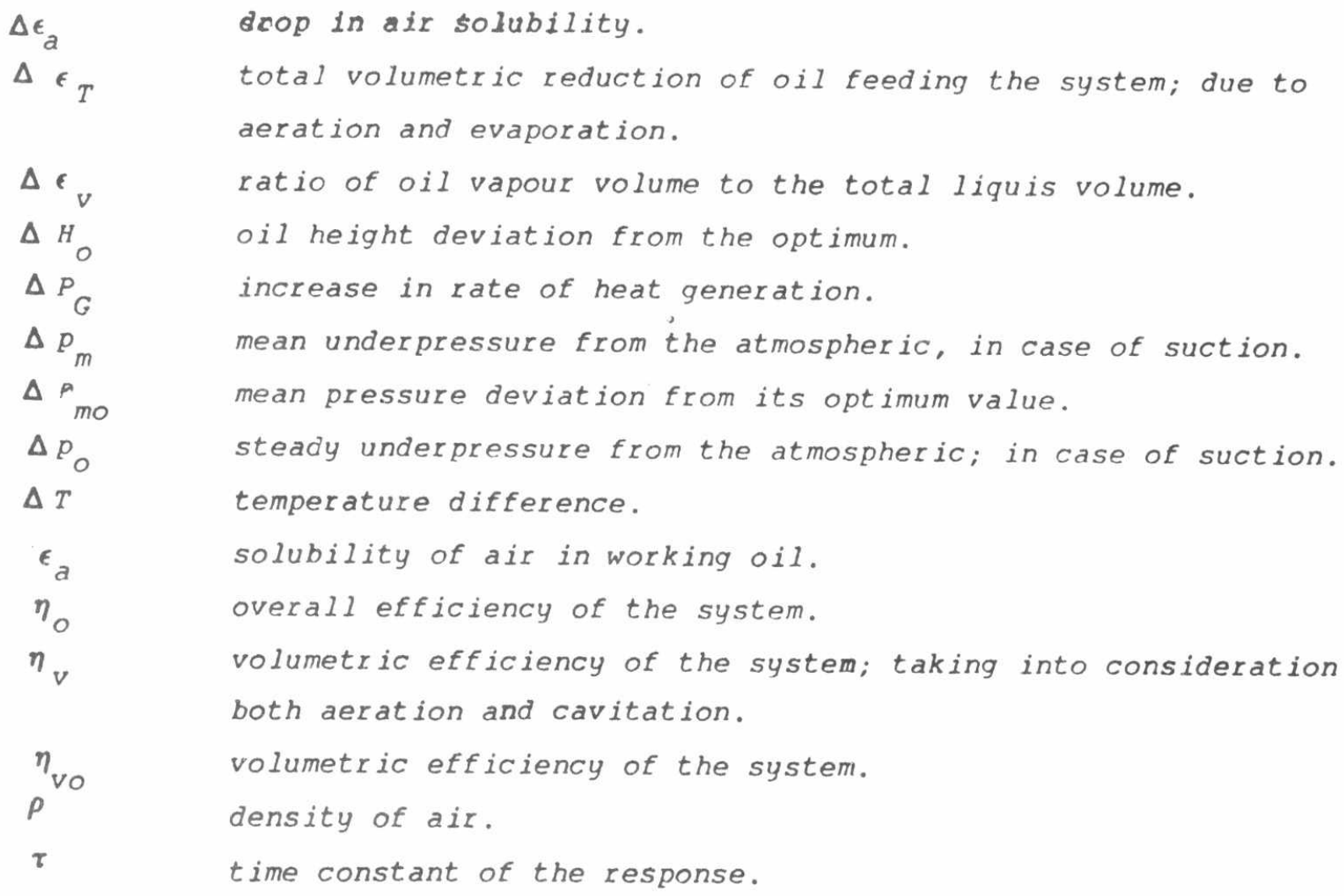

